\title{
Therapeutic Benefit of Placentrex in the Management of Acute Chemo Radiation Induced Mucositis in Oral Cancer Patients
}

\author{
Satish Srinivas Kondaveeti ${ }^{1}$, Divyambika $C \mathbf{V}^{2 *}$, Christopher John ${ }^{1}$, \\ Manickavasagam $\mathbf{M}^{3}$, Rajendiran $\mathrm{S}^{4}$
}

\begin{abstract}
Background: A troublesome and usually unavoidable consequence of Head and Neck chemo radiation is oral mucositis which decreases patients' compliance and negatively influences the outcome of therapy by increasing overall treatment time. Currently, no single effective recommended treatment exists for this problem and a variety of supportive care measures have been practiced with limited benefits. This study was done to evaluate the therapeutic benefit of Placentrex in the management of oral mucositis seen in oral cancer patients undergoing treatment with concurrent chemoradiation. Methodology: This study was carried out, as a retrospective analysis, on oral cancer patients undergoing concurrent chemoradiation with weekly Cisplatin regimen treated between Oct 2015 and July 2017. All the patients received $2 \mathrm{ml}$ of Inj Placentrex, once daily administered intramuscularly for 4 weeks, NSAIDs, topical analgesics, and mouth wash as treatment for oral mucositis. The results were compared with a historical control group of 40 oral cancer patients who had received treatment prior to the study period without receiving Inj Placentrex as a part of oral mucositis management. Results: Over $60 \%$ of the patients in both groups were older than 60 years of age. Buccal mucosa was the predominant sub site of the investigated cancer type. The addition of placentrex resulted in delay in the progression of mucositis, reduction of treatment breaks, regression of pain, and improvement of dysphagia while leading to no adverse effects $(\mathrm{p}<0.05)$. Conclusion: Placentrex appears to be a beneficial therapeutic option for the management of concurrent chemo-radiation induced acute oral mucositis in oral cancer patients.
\end{abstract}

Keywords: Chemoradiation- dysphagia- mucositis- placentrex- head and neck cancer

Asian Pac J Cancer Prev, 19 (11), 3099-3103

\section{Introduction}

One of the common cancers affecting both men and women in our country is head and neck cancer. Annually, two lakh new head and neck cancers are detected in India. On an average, more than two thirds of patients with head and neck cancers are present in advanced stage, where primary radiotherapy or postoperative chemoradiotherapy remains the most important treatment modality for these cancers (Kulkarni, 2013). Presently the standard of care in the management of unresectable squamous cell cancers of the head and neck is concurrent chemo radiation with high dose triweekly cisplatin $\left(\right.$ dose of $\left.100 \mathrm{mg} / \mathrm{m}^{2}\right)$. An alternative approach in cisplatin based concurrent chemoradiation is to administer the drug weekly at a dose of $40 \mathrm{mg} / \mathrm{m}^{2}$ concurrently with radiation (Geeta et al., 2006). The latter schedule has similar 2 year and 3 year Overall Survival (OS) rates but inferior 5 year OS rates in comparison to the high dose schedule (Chan et al., 2005). One of the important drawbacks of the weekly schedule is the higher incidence of grade 3 oral and pharyngeal mucositis (Guan et al., 2016). In its training module, the National Cancer Institute describes oral mucositis as "the reactive inflammation of the oral and oropharyngeal mucous membrane during radiotherapy or chemoradiotherapy of head and neck region". Studies have shown that $90 \%$ of patients undergoing cisplatin-based chemoradiation develop severe oral mucositis, affecting patients' compliance with planned treatment schedule (Bonomi and Batt, 2015). Accordingly, patients' compliance with therapy decreases, leading to treatment disruption, increase of overall treatment time which in turn negatively influences the treatment outcome (Guan et al., 2016).

Current strategies used in managing oral mucositis include - maintenance of good oral hygiene, maintenance of nutritional support (Zahn et al., 2012), regular use of oral rinses containing topical anesthetics such as lidocaine (Rastogi et al., 2015), or topical analgesics such as benzydamine (Kazemian et al., 2009). Other treatment strategies under evaluation to prevent oral mucositis include the use of Granulocyte-macrophage

${ }^{1}$ Department of Radiation Oncology, ${ }^{2}$ Faculty of Dental Sciences, ${ }^{3}$ Department of Medical Oncology, ${ }^{4}$ Department of Pathology, Sri Ramachandra Medical College and Research Institute, Porur, Chennai, India.*For Correspondence: cvdivyambika@, sriramachandra.edu.in 
colony-stimulating factor (GM-CSF) (Nidhi et al.,2005), Oral Glutamine (Chattopadhyay et al., 2014), Keratinocyte GrowthFactor-2 (KGF-2) (Le et al., 2011), and Amifostine (Vacha et al., 2003).

Despite the availability of many agents which are claimed to be beneficial in the management of radiation and chemotherapy related mucositis, their therapeutic benefit has not been shown to be consistent in clinical practice. As a result, oral mucositis remains an important obstacle which decreases the treatment compliance, negatively influencing the outcome of treatment. In spite of research work spanning several decades, there exists no single intervention currently that can completely prevent or treat oral mucositis. Nevertheless, one of the agents tried in our center for the management of oral mucositis was placentrex or human placental extract. Placentrex, a proprietary drug of $\mathrm{M} / \mathrm{S}$ Albert David Limited, is a purified, sterile, aqueous extract of human placenta that is thought to have anti-inflammatory and wound healing effect (Singh et al., 2015). Placental extract when administered into the sub mucosa of the oral cavity was shown to improve trismus and decrease theseverity offibrosis in patients with oral submucosal fibrosis (Gupta et al., 2012; Singh et al., 2015; Shah et al., 2016). Placental extract has been used for its analgesic and anti-inflammatory benefits in pelvic inflammatory disease (Prameela and Sharma, 2016). Topical application of placental extract solution on radiation induced cutaneous and perineal reactions could be beneficial (Kaul et al., 2001). By virtue of its anti-inflammatory, analgesic and epithelial growth promoting characteristics, it was useful when used as an intra muscular injection in acute radiation induced mucositis, in head and neck cancer patients undergoing radiotherapy (Kaushal et al., 2001). However, literature evidence on the benefit of placentrex in the setting of concurrent chemoradiation for head and neck cancers is sparse.

\section{Objective}

To evaluate the therapeutic effect of human placental extract in the management of chemo radiation induced mucositis in oral cancer patients.

\section{Materials and Methods}

This study was carried out, as a retrospective analysis, on 40 patients with squamous cell carcinoma of the oral cavity who underwent cisplatin-based concurrent chemo-radiation between Oct 2015 and June 2017. The results were compared with a historical control group of 40 oral cancer patients who had received treatment prior to the study period with the same chemo radiation protocol but who had not received Inj Placentrex as a part of management of oral mucositis. While the chemotherapy and supportive care for mucositis, including Inj Placentrex was administered in the Oncology Department, SRMC (Sri Ramachandra Medical College and Research Institute, the radiotherapy treatment was planned and delivered at a nearby private center under the supervision of the Radiation Oncologist of SRMC. Study participants
Data of 40 patients between 60 - 70 yrs of age with Karnofsky performance score of at least 80 with pathological confirmation of squamous cell carcinoma who received concurrent chemo radiation treatment with weekly Inj cisplatin during the study period was analyzed in the study. Patients were excluded if they were planned to receive chemo radiation with tri-weekly high dose cisplatin $\left(100 \mathrm{mg} / \mathrm{m}^{2}\right)$ or radiation. alone (curative, adjuvant or palliative and altered fractionated radiotherapy), they had cancer histology other than squamous cell carcinoma or recurrent disease, they had distant metastasis. Data of another 40 cancer patients meeting the same inclusion and exclusion criteria and treated with concurrent chemoradiation with weekly cisplatin prior to the period of study was taken as historical control data.

\section{Treatment protocol}

Radiation treatment provided for primary disease was External Beam Radiation using 6 MV Linac X ray photons with 3 Dimensional Conformal Radiotherapy (3DCRT) technique or IMRT technique at a dose of 66 Gy at 200 cGy per fraction in 33 fractions in 6.5 weeks with $6 \mathrm{MV}$ $\mathrm{X}$ ray photons. The chemotherapy protocol consisted of Inj cisplatin at a flat dose of $40 \mathrm{mg}$ given as intravenous infusion concurrently with weekly radiation on Mondays with appropriate antiemetic medicines and hydration. The patients with oral mucositis in the study group received $2 \mathrm{ml}$ of Human Placental Extract commercially available as Injection Placentrex manufactured by $\mathrm{M} / \mathrm{s}$ Albert David) once a day via deep intramuscular route from commencement of symptom till the end of therapy (on radiation treatment and non treatment days) while others in the historical control group received best supportive cares such as NSAIDS with Aceclofenac and Paracetamol combination, oral benzydamine mouth rinse, saline gargle, oral fluconazole tablets ( $200 \mathrm{mg}$ once daily for 14 days) for treatment of oral mucositis. The oral mucositis treatment was started at the commencement of the symptom, usually around the $10^{\text {th }}$ fraction of Radiotherapy.

\section{Data analysis}

The benefit of placentrex based supportive therapy for oral mucositis was assessed with respect to severity of oral mucositis (based on Radiation Therapy Oncology Group (RTOG) mucositis grading score) and severity of dysphagia using Functional Impairment Scale (FIS) which had been recorded in the treatment chart during patients' weekly review . In addition, overall treatment time (OTT) and time taken for complete healing of mucositis following treatment were the parameters used to assess the benefit.

\section{Results}

Table 1 shows the characteristics of patients included in this study. More than $50 \%$ of the patients analyzed in both groups were above 60 years of age with a preponderance of male sex. Most of the patients habituated to tobacco. Majority of the patients (above 85\%) in both groups had locally advanced oral cancers (stage III disease and higher). The anatomical site of cancer is given in Table 2 . 
Table 1. Patients' Characteristic

\begin{tabular}{|c|c|c|c|c|c|}
\hline \multirow[t]{2}{*}{ S. No } & \multirow[t]{2}{*}{ Characteristics } & \multicolumn{2}{|c|}{ Control Group } & \multicolumn{2}{|c|}{ Experimental Group } \\
\hline & & Frequency & Percentage & Frequency & Percentage \\
\hline \multirow[t]{3}{*}{1} & Age (in years) & & & & \\
\hline & $51-60$ & 14 & 35 & 19 & 47.5 \\
\hline & $>60$ & 26 & 65 & 21 & 52.5 \\
\hline \multirow[t]{3}{*}{2} & Sex & & & & \\
\hline & Male & 32 & 80 & 33 & 82.5 \\
\hline & Female & 8 & 20 & 7 & 17.5 \\
\hline \multirow[t]{3}{*}{3} & Tobacco use & & & & \\
\hline & Present & 29 & 72.5 & 36 & 90 \\
\hline & Absent & 11 & 27.5 & 4 & 10 \\
\hline \multirow[t]{5}{*}{4} & Disease Stage & & & & \\
\hline & Stage II & 4 & 10 & 5 & 12.5 \\
\hline & Stage III & 13 & 32.5 & 15 & 37.5 \\
\hline & Stage IVa & 17 & 42.5 & 12 & 30 \\
\hline & Stage IV b & 6 & 15 & 8 & 20 \\
\hline
\end{tabular}

Table 2. Anatomical Site of Malignancy Among the Patients

\begin{tabular}{lccccc}
\hline S. No & Anatomical site of Cancer & \multicolumn{2}{c}{ Control group } & \multicolumn{2}{c}{ Study Group } \\
& & Frequency & Percentage & Frequency & Percentage \\
\hline 1 & Buccal Mucosa (cheek) & 12 & 30 & 18 & 45 \\
2 & Anterior Tongue & 9 & 22.5 & 8 & 20 \\
3 & Gingiva & 7 & 17.5 & 5 & 12.5 \\
4 & Retromolar trigone & 4 & 10 & 4 & 10 \\
5 & Floor of Mouth & 5 & 12.5 & 3 & 7.5 \\
6 & Hard Palate & 3 & 7.5 & 2 & 5 \\
\hline
\end{tabular}

In our study, the predominant cancer site was buccal mucosa.

\section{Received treatment}

The median number of weekly Cisplatin chemotherapy cycles received by patients in both groups was 4 wherein 8 patients $(20 \%)$ in the control group and 9 patients $(22.5 \%)$ in the study group received at least 5 cycles. None of the patients tolerated more than 5 cycles of weekly Cisplatin (median of 4 cycles). Patients were given growth factor support with Inj GCSF 300 micrograms sc. OD as well as prophylactic oral antibiotics were administered when the absolute neutrophil count was lesser than 1500 . None of the patients required platelet transfusion or experienced treatment break due to febrile neutropenia. Patients with grade III dysphagia were fed through nasogastric tube until the end of therapy. All the patients completed the planned RT protocol of 66Gy in 33 \#. Patients with oral lesions such as tongue and floor of mouth lesions, in whom bilateral neck irradiation was considered, received IMRT technique with sparing of the parotid.

Each patients with unilateral lesions received 3D conformal treatment. Patients who were treated until January 2017 received treatment with $6 \mathrm{MV}$ photons from Varian DBX linear accelerator but patients who were treated after January 2017 received RT with 6 MV photons from Versa HD linear accelerator.

Detailed analysis of data was performed regarding weekly grade of mucositis and grade of dysphagia, overall treatment time, and time for recovery of mucositis. The same parameters were recorded from the treatment charts and case sheets of the patients included in the control group. The data on the two arms were compared based

Table 3. Comparative Analysis between Study and Historical Control Groups

\begin{tabular}{lccccc}
\hline S. No & Treatment Efficacy & $\begin{array}{c}\text { Historical Control } \\
\text { Group }(\%) \mathrm{n}=40\end{array}$ & $\begin{array}{c}\text { Study } \\
\text { Group (\%) } \mathrm{n}=40\end{array}$ & chi square value & p value \\
\hline 1 & Progression to grade 3 mucositis or higher & $32(80 \%)$ & $12(30 \%)$ & 5.617 & 0.017 \\
2 & Progression to grade 3 dysphagia or higher & $32(80 \%)$ & $12(30 \%)$ & 5.617 & 0.017 \\
3 & Patient compliance with RT (OTT $\leq$ 50 days) & $27(67.5 \%)$ & $34(85 \%)$ & 1.69 & 0.193 \\
4 & Healing within 2 weeks & $4(10 \%)$ & $23(57.5 \%)$ & 10.09 & 0.001 \\
\hline
\end{tabular}


on incidence of grade III mucositis, grade III dysphagia, time taken to complete the treatment protocol (Overall Treatment Time), and incidence of persistent mucositis 2 weeks post treatment.

\section{Discussion}

Human placenta has been described as an immunologically privileged organ (Vineeta et al., 2016) and its therapeutic effect was investigated first by Russian ophthalmologist, Filatov, who described the placental extract as a biogenic stimulator which could promote recovery of diseased tissues. Placental possess analgesic, wound healing, and anti-inflammatory properties. Various biological products such as glycosaminoglycans, nucleic acids, polydeoxyribonucleotides, hormones, and proteins have been isolated from the placenta, suggesting its therapeutic potential as a wound healing agent (Chakraborthy and Bhattacharya, 2012; Vineeta et al., 2016). The aqueous extract of human placenta marketed as placentrex has been shown to possess several wound healing properties and exhibits tissue regeneration in chronic and non healing wounds induced by inherent pathologies like diabetes. It is extremely effective in healing wounds as it increases the blood supply in tissues (Anil and Beena, 1993) and enhance regeneration and recovery of the tissue (Khanna and Andrade, 1995). Its immunomodulatory effect lies in restoring $T$ and B lymphocytes dysfunction to normal (Ansari et al., 1994). Placental extract contains bioactive peptides and amino acids which facilitate wound healing. It is also hypothesized that the anti-inflammatory property of placentrex is facilitated by the suppression of prostaglandin synthesis and perotonin release (Sur et al., 2003). It also possesses anti-inflammatory and anti-platelet aggregation properties. These properties along with histopathological evidence show that accumulation of collagen fibrils and epithelialization could be the underlying pathophysiology involved in the therapeutic benefit of human placental extract (Chakraborty and Bhattacharya, 2012).

Chemotherapy or radiation-induced oral mucositis is seen in over $80 \%$ of the patients with head and neck tumor irradiation. Though several conventional treatment modalities are available, none of them have been proven to be efficient. Our study highlighted the therapeutic outcomes of human placental extract when used in the management of chemotherapy or radiation- induced oral mucositis. In our study, the administration of placentrex along with conventional treatment, in the form of soda bicarbonate mouthwash along with Disprin gargle and $2 \%$ viscous xylocaine, for the patients undergoing concurrent chemoradiation for head and neck cancers helped to reduce the intensity of mucositis, improve symptoms such as dysphagia, and reduce pain and healing time all of which resulted in increased patients' compliance with the planned treatment schedule. In patients who received placentrex injection, the progression of oral mucositis to grade III and above reduced from $80 \%$ to $30 \%$. A similar observation was also seen with respect to dysphagia severity. Nearly $85 \%$ of the patients who received placentrex injection completed the treatment protocol within 50 days (against $67.5 \%$ in the control group). In $58 \%$ of patients who received placentrex, the mucositis resolved within two weeks after treatment. The findings of our study were in concurrence with a study done by Kaushal et al., (2001) who analyzed the efficacy of placentrex in treating mucositis induced by radiation alone in head and neck cancer patients. Another important point observed here was that none of the patients developed any allergy or side effect or biochemical derangement after placentrex administration, indicating the favorable safety profile of the human placental extract.

However, this study had a number of limitations; for instance, it was retrospective and historical control groups were unevenly matched with respect to disease stage and site. The other limitation was related to number of chemotherapy cycles received (4 versus 5 cycles) by various patients, which could influence the severity of mucositis. Variations were also observed in used RT technique (3D Conformal RT versus Intensity Modulated RT) where lower normal tissue side effects induced by the latter technique could also influence the outcome. Thus in conclusion the need of the hour would indeed be a planned double blind Prospective Randomised Control trial on concurrent chemoradiation induced oral mucositis with significant statistical power to verify this preliminary data suggesting benefit with Placentrex.

In conclusion, chemotherapy or radiation-induced oral mucositis delimits the patient's treatment response in head and neck cancers. The existing conventional treatments for radiation and chemotherapy-induced mucositis do not provide a comprehensive solution for this troublesome but inevitable side effect of cancer treatment. This study demonstrated that placentrex with known tissue healing and regenerative properties can be considered in combination with other supportive care measures to overcome the problem of chemotherapy-induced mucositis thereby improve patients' chemoradiation to treatment.

\section{References}

Anil S, Beena VT (1993). Oral submucous fibrosis in a 12- year - old girl: case report. Pediatr Dent, 16, 120-2.

Ansari KU, Nira G, Bapat SK, et al (1994). An experimental and clinical evaluation of immuno-modulating potential of human placental extract. Indian J Pharmacol, 26, 130 - 2.

Chan AT, Leung SF, Ngan RK, et al (2005). Overall survival after concurrent cisplatin-radiotherapy compared with radiotherapy alone in locoregionally advanced nasopharyngeal carcinoma. J Natl Cancer Inst, 97, 536-9.

Chattopadhyay S, Saha A, Azam M, Mukherjee A, Sur PK (2014). Role of oral glutamine in alleviation and prevention of radiation-induced oral mucositis: A prospective randomized study. South Asian J Cancer, 3, 8-12.

Geeta SN, Padmanabhan TK, Samuel J, et al (2006). Comparison of acute toxicities of two chemotherapy schedules for head and neck cancers. J Cancer Res Ther, 2, 100-4.

Gupta J, Shrinivasan SV, Daniel JM (2012). Effiacy of betamethasone, placental extract and hyaluronidase in the treatment of OSMF: a comparative study. E J Dent, 2, 132-3

Jian G, Yue Z, Qinyang L, et al (2016). A meta-analysis of weekly cisplatin versus three weekly cisplatin chemotherapy plus concurrent radiotherapy (CRT) for advanced head and 
neck cancer (HNC). Oncotarget, 7, 185-93.

Katharia SK (1992). The effects of placenta extract in management of OSMF. Indian J Pharmacol, 24, 181-3.

Kaul R, Chaudhary V, Mukhopadayay P (2001). To evaluate the effect of local placentrex therapy in reducing side effects of radiation in patients of cervical carcinoma. Antiseptic, 98, 131-2.

Kaushal V, Verma K, Manocha S, Hooda HS , Das BP (2001). Clinical evaluation of placentrex in radiation induced oral mucositis. Int $J$ Tissue React, 13, 105-10.

Kazemian A, Kamian S, Aghili M, Hashemi FA, Haddad P (2009). Benzydamine for prophylaxis of radiation-induced oral mucositis in head and neck cancers: A double-blind placebo-controlled randomized clinical trial. Eur J Cancer Care (Engl), 18, 174-8.

Le QT, Kim HE, Schneider CJ, et al (2011). Palifermin reduces severe mucositis in definitive chemoradiotherapy of locally advanced head and neck cancer: A randomized, placebo-controlled study. J Clin Oncol, 29, 8-14.

Manik Rao K (2013). Head and neck cancer burden in India. Int J Head Neck Surg, 4, 29-35.

Marcelo B, Katharine B (2015). Supportive management of mucositis and metabolic derangements in head and neck cancer patients. Cancers (Basel), 7, 43-57.

National Cancer Institute. SEER training modules. Cancer as a disease. Available from http:// training .seer.cancer.gov/ module cancer disease / unit 2 whats cancerl definition.html

Nidhi PS, Bapna A (2005). The optimal use of granulocyte macrophage colony stimulating factor in radiation induced mucositis in head and neck squamous cell carcinoma. J Cancer Res Ther, 1, 136 -41.

Palak HS, Rashmi V, Chandramani BM, Vaishnavee V (2016). Therapeutic efficacy of two treatment regimen for oral submucous fibrosis. J Clin Diag Res, 10, 63-4.

Piyali DC, Debasish B (2012). Aqueous extract of human placenta as a therapeutic agent. Recent advances in research on the human placenta. Dr. Jing Zheng (Ed.), ISBN: 978-953-51-0194-9, InTech, Available from: http://www. intechopen.com/books/recent-advances-in-research-on-thehumanplacenta/aqueous-extract-of-human-placenta-as-atherapeutic-agent.

Prameela KDS (2016). Clinical efficacy of placentrex injection in pelvic inflammatory disease. Indian J Obstet Gynaecol Res, 3, 65-7

Singh DT, Padshetty S, Shreen S, et al (2015). Injection of placentrex in the management of oral submucous fibrosis. Int J Mod Sci Eng Technol, 2, 23-30.

Sur TK, Biswas TK, Ali L, Mukherjee B (2003) Anti-inflammatory and anti-platelet aggregation activity of human placental extract. Acta Pharmacol Sin, 24, 187-92.

Trisha R, Sunil KK, V Naresh, Rohit KS (2015). Preventive strategies in management of oral mucositis. IJTOH, 1, 9-13.

Vacha P, Fehlauer F, Mahlmann B, et al (2003). Randomized phase III trial of postoperative radiochemotherapy $+/$ amifostine in head and neck cancer. Is there evidence for radioprotection?. Strahlenther Onkol, 179, 385-9.

Vineeta G, Aditya S, Jithendra KD, et al (2016). Placental extract -the magical wound healer, Next milestone in the healing of periodontal surgery. IOSR J Dent Med Sci (IOSR-JDMS), 15, 73-9

Zahn KL, Wong G, Bedrick EJ, et al (2012). Relationship of protein and calorie intake to the severity of oral mucositis in patients with head and neck cancer receiving radiation therapy. Head Neck, 34, 655-62.
Placentrex in Chemo Radiation Induced Oral Mucositis

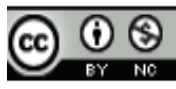

This work is licensed under a Creative Commons AttributionNon Commercial 4.0 International License. 